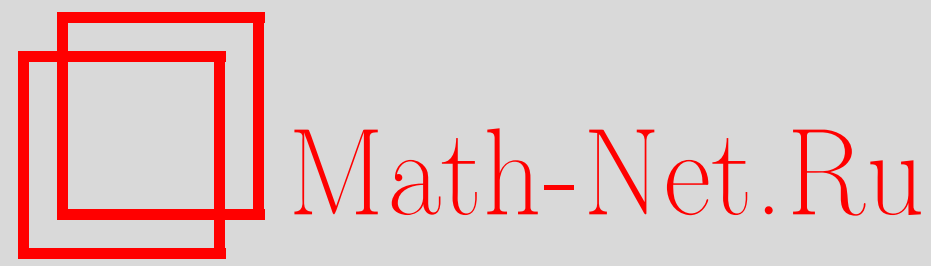

И. А. Кузин, Об отсутствии счетного множества решений у некоторых эллиптических задач с правой частью и суперкритическим показателем нелинейности, Матем. заметки, 1996, том 60, выпуск 3, 452-457

DOI: https://doi.org/10.4213/mzm1849

Использование Общероссийского математического портала MathNet.Ru подразумевает, что вы прочитали и согласны с пользовательским соглашением

http://www.mathnet.ru/rus/agreement

Параметры загрузки:

IP : 35.174 .16 .151

26 апреля 2023 г., 14:53:21

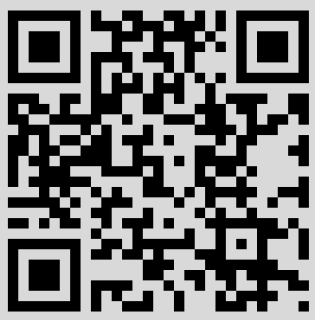




\section{ОБ ОТСУТСТВИИ СЧЕТНОГО МНОЖЕСТВА РЕШЕНИЙ У НЕКОТОРЫХ ЭЛЛИПТИЧЕСКИХ ЗАДАЧ С ПРАВОЙ ЧАСТЬЮ И СУПЕРКРИТИЧЕСКИМ ПОКАЗАТЕЛЕМ НЕЛИНЕЙНОСТИ}

\section{И.А. Кузин}

Формулировка результатов. В данной работе мы рассматриваем "антикоэрцитивную" задачу

$$
\begin{aligned}
& \Delta u+|u|^{p-1} u=h(\mathbf{x}) \quad \text { в } \Omega, \\
& u(\partial \Omega)=0,
\end{aligned}
$$

где $\Omega$ - гладкая ограниченная область в $\mathbb{R}^{N}, N>2, p+1 \geqslant 2^{*}, 2^{*} \equiv 2 N /(N-2)$ критический показатель нелинейности.

Известно, что если $h \equiv 0$, то задача (1) имеет при $2<p+1<2^{*}$ счетное множество различных решений из $H_{1}^{0}(\Omega)$, если же $p+1 \geqslant 2^{*}$ и область $\Omega$ звездная (например, шар), то задача (1) c $h \equiv 0$ не имеет решений, отличных от тривиального (С. И. Похожаев [1]).

Интерес представляет случай нетривиальных правых частей. Известно, что если $2<p+1<(2 N-2) /(N-2)$, то для любой функции $h \in L_{2}(\Omega)$ задача (1) имеет счетное множество решений из $H_{1}^{0}(\Omega)$ (А. Бари, П.-Л. Лионс [2], М. Стуве [3], А. Бари, Х. Берестицкий [4]).

Возникает закономерный вопрос о существовании решений для критического и суперкритического случаев $p+1 \geqslant 2 *$ при нетривиальной правой части.

Исследуем этот вопрос, когда $\Omega$ - шар и $h$ - радиальная функция.

В работах С.И. Похожаева (см., например, [5]) приведены некоторые достаточные условия на $h$, при которых радиальная задача (1) не имеет положительных или отрицательных решений.

Мы покажем, что для многих $h$ высокой гладкости задача (1) в критическом или суперкритическом случаях, в отличие от докритического случая, не допускает счетного множества радиальных решений.

Итак, в радиальном случае и случае нечетных $p$ задача (1) представима в виде

$$
\begin{aligned}
& u^{\prime \prime}+\frac{N-1}{r} u^{\prime}+|u|^{p-1} u=h(r), \quad 0 \leqslant r \leqslant R \\
& u^{\prime}(0)=0, \quad u(R)=0
\end{aligned}
$$

где $r=|\mathbf{x}|$, а $R>0$ есть радиус шара $\Omega$.

$\mathrm{C}$ целью изучения задачи (2) на предмет существования или несуществования решений мы будем исследовать вспомогательную задачу Коши

$$
\begin{aligned}
& u^{\prime \prime}+\frac{N-1}{r} u^{\prime}+|u|^{p-1} u=h(r), \quad 0 \leqslant r<+\infty \\
& u^{\prime}(0)=0, \quad u(0)=a
\end{aligned}
$$

Работа выполнена при частичной поддержке Российского фонда фундаментальных исс ледований, грант № 93-011-197, и Международного научного фонда, гранты NMT000, NMT300. 
при разных значениях $a$. Стандартные рассуждения показывают, что если $h \in$ $C_{\mathrm{loc}}([0,+\infty))$, то задача (3) для каждого $a$ имеет единственное решение из $C_{\mathrm{loc}}^{2}([0,+\infty))$, которое мы будем обозначать $u_{a}$.

Аналогично, решение задачи

$$
\begin{aligned}
& u^{\prime \prime}+\frac{N-1}{r} u^{\prime}+|u|^{p-1} u=h(r), \quad R \leqslant r<+\infty, \\
& u(R)=a, \quad u^{\prime}(R)=b
\end{aligned}
$$

будем обозначать $u_{R, a, b}$. Для $p+1>2 *$ обозначим также

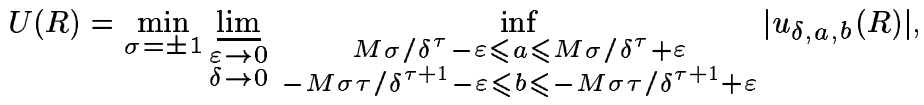

где $\tau=2 /(p-1), M=(\tau(N-\tau-2))^{\tau / 2}$.

Будем считать, что $h$ - действительная радиальная голоморфная функция на некотором открытом шаре $B_{R+\varepsilon}$.

Основной результат следующий.

Tеорема 1. Пусть $p+1 \geqslant 2^{*}$ - челое нечетное число, $h$ как описано выше. Пусть $u_{0}(R) \neq 0$ при $p+1=2^{*} u U(R) \neq 0$ npu $p+1>2^{*}$. Тогда задача (2) имеет не более конечного числа классических решений $u \in C^{2}([0, R)) \cap C([0, R])$.

Доказательство. Покажем, как теорема 1 доказьвается для случая $p+1=2^{*}$. Случай $p+1>2^{*}$ аналогичен.

Тогда теорема 1 основьвается на следующем результате.

Teоpema 2. Пусть $p+1=2^{*}, h \in C_{\text {loc }}([0, \infty)) \quad u \quad u_{0}(R) \neq 0 . \quad$ Пусть для любого а а существует не более конечного числа значений $|a| \leqslant a_{0}$, при которых решение $u_{a}$ задачи (3) является решением задачи (2). Тогда задача (2) имеет не более конечного числа классических решений $u \in C^{2}([0, R)) \cap C([0, R])$.

Теорема 2 в свою очередь основьвается на следующей лемме.

ЛЕмма 1. Пусть $p+1=2^{*}, h \in C_{\mathrm{loc}}([0, \infty))$. Тогда для любьх $0<r_{0} \leqslant$ $R_{0}<\infty$

$$
\lim _{|a| \rightarrow \infty} \max _{r_{0} \leqslant r \leqslant R_{0}}\left|u_{a}(r)-u_{0}(r)\right|+\left|u_{a}^{\prime}(r)-u_{0}^{\prime}(r)\right|=0 .
$$

Представим на время, что лемма 1 верна, и докажем теоремы 2 и 1.

ДОКАЗАТЕЛЬСТВО ТЕОРЕМЫ 2 . В силу предположения $u_{0}(R) \neq 0$. Тогда вследствие леммы $1 u_{a}(R) \neq 0$ для достаточно больших $|a|$. Но согласно условию теоремы, коль скоро выбрано некоторое $a_{0}$, существует не более конечного числа значений $|a| \leqslant a_{0}$, при которых решение $u_{a}$ задачи (3) является решением задачи (2). 
ДоКАЗАТЕЛЬСТво ТЕОРЕмЫ 1. Для нечетных $p$ функция $u^{p}$ голоморфна. В силу голоморфности $h$ для любых конечных $a_{0}>0$ функция $u .(R)$, рассмотренная на отрезке $\left[-a_{0}, a_{0}\right]$, не может иметь бесконечного числа нулей. В противном случае $u \cdot(R) \equiv 0$ (см., например, [6], [7]), что не так хотя бы потому, что $u_{0}(R) \neq 0$. Следовательно, мы можем применить теорему 2.

Теорема 1 доказана.

Далее для удобства обозначений мы ограничимся рассмотрением случая $a>0$, $p+1=2^{*}$ - нечетное целое, всегда так полагая и часто не оговаривая это особо.

Итак, нам осталось доказать лемму 1. Для этого вместе с задачей (3) рассмотрим также следующую вспомогательную задачу

$$
\begin{aligned}
& v^{\prime \prime}+\frac{N-1}{r} v^{\prime}+v^{p}=0, \quad 0 \leqslant r<+\infty \\
& v^{\prime}(0)=0, \quad v(0)=a .
\end{aligned}
$$

Решение этой задачи с заданным $a$ будем обозначать $v_{a}$. Очевидно, что $v_{a} \in$ $C_{\mathrm{loc}}^{2}([0,+\infty))$.

ЛЕмма 2. Для любъх $0<r_{0} \leqslant R_{0}<\infty$

$$
\lim _{|a| \rightarrow \infty} \max _{r_{0} \leqslant r \leqslant R_{0}}\left|v_{a}(r)\right|+\left|v_{a}^{\prime}(r)\right|=0 .
$$

ДокАЗАТЕЛЬСтво. Можно показать (см., например, [8]), что для $p+1=2^{*}$

$$
v_{a}(r)=a v_{1}\left(|a|^{(p-1) / 2} r\right)
$$

где

$$
\lim _{r \rightarrow \infty}\left(\left|v_{1}(r)\right|+\left|v_{1}^{\prime}(r)\right|\right) r^{2 /(p-1)}=0 .
$$

Из (5) и (6) следует утверждение леммы.

ЗАмеч АниЕ. Хорошо известно (см., например, [8]), что $v_{1}>0$ и $v_{1}^{\prime} \leqslant 0$.

В основе доказательства леммы 1 лежит следующее наблюдение.

Лемма 3. Для любого $\varepsilon>0$ сущ,ествует $a_{0}>0$ maкое, ито для всех $a \geqslant a_{0}$

$$
\max _{\left\{0 \leqslant r<+\infty: v_{a}(r) \geqslant a_{0}\right\}}\left(\left|u_{a}(r)-v_{a}(r)\right|^{2}+\left|u_{a}^{\prime}(r)-v_{a}^{\prime}(r)\right|^{2}\right) \leqslant \varepsilon .
$$

ДОКАЗАТЕЛЬСтво. Обозначим $w=u_{a}-v_{a}$. Тогда

$$
\begin{aligned}
& w^{\prime \prime}+\frac{N-1}{r} w^{\prime}+\left(v_{a}+w\right)^{p}-v_{a}^{p}=h(r), \quad 0 \leqslant r<+\infty \\
& w(0)=0, \quad w^{\prime}(0)=0
\end{aligned}
$$

Заметим, что $\left(v_{a}+w\right)^{p}-v_{a}^{p}=f\left(v_{a}, w\right) w^{2}+p v_{a}^{p-1} w$, где

$$
\lim _{|t| \rightarrow \infty} \max _{|s| \leqslant 1} f(t, s) t^{1-p}=0 .
$$


Домножим уравнение (8) на $w^{\prime}$ и проинтегрируем от 0 до $R$ с учетом начальных условий. Получим

$$
\begin{aligned}
\frac{1}{2}\left(\left(w^{\prime}\right)^{2}+p v_{a}^{p-1} w^{2}\right)(R)= & -(N-1) \int_{0}^{R} \frac{\left(w^{\prime}\right)^{2}}{r} d r+\frac{p}{2} \int_{0}^{R}\left(v_{a}^{p-1}\right)^{\prime} w^{2} d r \\
& -\int_{0}^{R} f\left(v_{a}, w\right) w^{2} w^{\prime} d r+\int_{0}^{R} h w^{\prime} d r
\end{aligned}
$$

Первые два слагаемые в правой части (10) неположительны. Для первого слагаемого это очевидно сразу. Второе же является таковым вследствие того, что $v_{a}>0$, $v_{a}^{\prime} \leqslant 0$.

Следовательно, будем иметь

$$
\frac{1}{2}\left(\left(w^{\prime}\right)^{2}+p v_{a}^{p-1} w^{2}\right)(R) \leqslant-\int_{0}^{R} f\left(v_{a}, w\right) w^{2} w^{\prime} d r+\int_{0}^{R} h w^{\prime} d r .
$$

Выберем некоторым образом $\varepsilon>0$. Не теряя общности, положим $\varepsilon \leqslant 1 / 2$. В силу начальных условий задачи (8), а также регулярности $v_{a}$ и $h$, для любого $a_{0}>0$ и каждого $a \geqslant a_{0}$ существует такое $\delta_{a}$ с $1 \geqslant \delta_{a}>0$, что

$$
\max _{\left\{0 \leqslant r \leqslant \delta_{a}: v_{a}(r) \geqslant a_{0}\right\}}\left(|w(r)|^{2}+\left|w^{\prime}(r)\right|^{2}\right) \leqslant 1 .
$$

Обозначим

$$
\begin{gathered}
h_{M}=\max _{|r| \leqslant 1}|h(r)|, \quad w_{R}^{\prime}=\max _{|r| \leqslant R}\left|w^{\prime}(r)\right|, \quad w_{R}=\max _{|r| \leqslant R}|w(r)|, \\
\left(w^{2} v_{a}^{p-1}\right)_{R}=\max _{|r| \leqslant R}\left(w^{2} v_{a}^{p-1}\right)(r) .
\end{gathered}
$$

Получим при $R \in\left\{0 \leqslant r \leqslant \delta_{a}: v_{a}(r) \geqslant a_{0}\right\}$ :

$$
\begin{aligned}
\left|\int_{0}^{R} h w^{\prime} d r\right| \leqslant h_{M} w_{R}^{\prime} R & \\
\left|\int_{0}^{R} f\left(v_{a}, w\right) w^{2} w^{\prime} d r\right| & \leqslant \frac{p}{2} \int_{0}^{R}\left|v_{a}^{p-1} w^{2} w^{\prime}\right| d r \\
& \leqslant \frac{p}{2}\left(w^{2} v_{a}^{p-1}\right)_{R} w_{R}^{\prime} R d r, \quad a_{0} \text { велико. }
\end{aligned}
$$

Вторая оценка (12) следует из (9) для достаточно больших $a_{0}$ в силу $\left|w_{R}\right| \leqslant 1$. Из (11), (12) следует

$$
\frac{1}{2}\left(w_{R}^{\prime}\right)^{2}+\frac{p}{2}\left(w^{2} v_{a}^{p-1}\right)_{R} \leqslant\left(h_{M}+\frac{p}{2}\left(w^{2} v_{a}^{p-1}\right)_{R}\right) w_{R}^{\prime} R .
$$

Следовательно, выбирая

$$
R_{1} \equiv \frac{\varepsilon / 2}{h_{M} / p+\varepsilon / 2}
$$

получим для $R \in\left\{0 \leqslant r \leqslant \min \left\{\delta_{a}, R_{1}\right\}: v_{a}(r) \geqslant a_{0}\right\}$ вследствие $\left|w_{R}^{\prime}\right| \leqslant 1$ :

$$
\left(w^{2} v_{a}^{p-1}\right)_{R} \leqslant \varepsilon .
$$


Вследствие $v_{a}(r) \geqslant a_{0}$ для рассматриваемых $r$ мы имеем из (14) при $a_{0} \geqslant 1$

$$
w_{R}^{2} \leqslant \varepsilon
$$

Сопоставляя (13) и (14), получим

$$
\frac{1}{2} w_{R}^{\prime} \leqslant\left(h_{M}+\frac{p}{2} \varepsilon\right) R,
$$

откуда для

$$
R=\min \left\{R_{2}, \delta_{a}\right\}, \quad R_{2}=\min \left\{R_{1}, \frac{1}{2 p}, \frac{\varepsilon}{4 h_{M}}\right\}
$$

следует

$$
w_{R}^{\prime 2} \leqslant \varepsilon
$$

Итак, если $\varepsilon \leqslant 1 / 2$, то, выбирая $R$ согласно (16), мы получим априорные оценки (15), (17). В силу выбора $\varepsilon$ из (15), (17) следует, в частности, что $\left|w_{R}\right|<1$, $\left|w_{R}^{\prime}\right|<1$. Значит, эти оценки верны на самом деле для $R=R_{2}$.

Поскольку в силу леммы $2\left\{0 \leqslant r<+\infty: v_{a}(r) \geqslant a_{0}\right\} \subset\left[0, R_{2}\right]$ для достаточно больших $a_{0}$, лемма 3 доказана.

Лемма 4. Для любого $\varepsilon>0$ существует такое $a_{1}>0$, ито для любого $a \geqslant a_{1}$ существует $R_{1} \leqslant \varepsilon$ такое, что

$$
\left|u_{a}\left(R_{1}\right)\right|+\left|u_{a}^{\prime}\left(R_{1}\right)\right| \leqslant 2 \varepsilon .
$$

ДоКАЗАТЕЛЬСтво. Выберем, не теряя общности, некоторое $0<\varepsilon \leqslant 1 / 2$. В силу лемм 2,3 для любого такого $\varepsilon$ существует $a_{0}>0$ такое, что для любого $a \geqslant a_{0}$ в точке $r_{0}$, в которой $v_{a}\left(r_{0}\right)=a_{0}$, выполнено

$$
\left|w\left(r_{0}\right)\right|+\left|w^{\prime}\left(r_{0}\right)\right| \leqslant \frac{\varepsilon}{2}, \quad r_{0} \leqslant \frac{\varepsilon}{2} .
$$

В силу уравнения (8)

$$
\begin{aligned}
w(R)= & \frac{1}{N-2} \int_{r_{0}}^{R}\left(v_{a}^{p}-\left(v_{a}+w\right)^{p}+h\right) r\left(1-\left(\frac{r}{R}\right)^{N-2}\right) d r \\
& +w^{\prime}\left(r_{0}\right) \int_{r_{0}}^{R} \frac{r_{0}^{N-1}}{r^{N-1}} d r+w\left(r_{0}\right) .
\end{aligned}
$$

Имеем в силу (19) для $1 \geqslant R>r_{0}$

$$
|w(R)| \leqslant \int_{r_{0}}^{R}\left|f\left(v_{a}, w\right) w^{2}+p v_{a}^{p-1} w\right| \frac{r}{N-2} d r+\frac{h_{M}}{2} \frac{R^{2}-r_{0}^{2}}{N-2}+\frac{\varepsilon}{2} .
$$

Следовательно, если $|w| \leqslant 1$, и $a_{0} \geqslant 1$ достаточно велико, то в силу (9) и $v_{a} \leqslant a_{0}$ при $1 \geqslant R>r_{0}$

$$
|w(R)| \leqslant \frac{2}{N-2} a_{0}^{p-1} p \int_{r_{0}}^{R} r d r+\frac{h_{M}}{2} \frac{R^{2}-r_{0}^{2}}{N-2}+\frac{\varepsilon}{2} .
$$

Иначе, для $1 \geqslant R_{0}>r_{0}$, для которых $|w| \leqslant 1$,

$$
\max _{r_{0} \leqslant R \leqslant R_{0}}|w(R)| \leqslant a_{0}^{p-1} p \frac{R_{0}^{2}-r_{0}^{2}}{N-2}+\frac{h_{M}}{2} \frac{R_{0}^{2}-r_{0}^{2}}{N-2}+\frac{\varepsilon}{2} .
$$


Выберем максимальное $R_{0} \leqslant 1$ среди тех, для которых выполнено

$$
\frac{R_{0}^{2}-r_{0}^{2}}{N-2} \leqslant \min \left\{\frac{\varepsilon}{4 p a_{0}^{p-1}}, \frac{\varepsilon}{2 h_{M}}\right\}, \quad R_{0}-r_{0} \leqslant \frac{\varepsilon}{2} .
$$

Тогда

$$
\max _{r_{0} \leqslant R \leqslant R_{0}}|w(R)| \leqslant \frac{\varepsilon}{4}+\frac{\varepsilon}{4}+\frac{\varepsilon}{2}=\varepsilon, \quad R_{0} \leqslant \varepsilon .
$$

Аналогичные рассуждения для производной и соответствующее переобозначение для $\varepsilon$ приводят для соответствующего $1 \geqslant R_{0}>r_{0}$ к оценке

$$
\max _{r_{0} \leqslant R \leqslant R_{0}}\left(\left|u_{a}(R)-v_{a}(R)\right|+\left|u_{a}^{\prime}(R)-v_{a}^{\prime}(R)\right|\right) \leqslant \varepsilon, \quad R_{0} \leqslant \varepsilon .
$$

Аналогично лемме 2 для любого $\varepsilon>0$ существует $a_{1} \geqslant a_{0}$ такое, что для каждого $a \geqslant a_{1}$ существует $R_{1}$ с $r_{0}<R_{1} \leqslant R_{0}$ и

$$
\left|v_{a}\left(R_{1}\right)\right|+\left|v_{a}^{\prime}\left(R_{1}\right)\right| \leqslant \frac{\varepsilon}{2} .
$$

Тогда из (20), (21) следует, что для достаточно больших $a_{1}$ выполнено (18).

ДоКАЗАТЕЛЬСТВА ЛЕМмы 1. В силу начальных данных для любого $\varepsilon>0$ существует такое $R$, что

$$
\max _{0 \leqslant r \leqslant R}\left|u_{0}(r)\right|+\left|u_{0}^{\prime}(r)\right| \leqslant \varepsilon .
$$

$\mathrm{C}$ другой стороны, в силу леммы 4 для любого $\varepsilon>0$ существует такое $a_{0}$, что для любого $a \geqslant a_{0}$ существует $r_{1} \leqslant R$ такое, что $\left|u_{a}\left(r_{1}\right)\right|+\left|u_{a}^{\prime}\left(r_{1}\right)\right| \leqslant \varepsilon$, т.е.

$$
\left|u_{0}\left(r_{1}\right)-u_{a}\left(r_{1}\right)\right|+\left|u_{0}^{\prime}\left(r_{1}\right)-u_{a}^{\prime}\left(r_{1}\right)\right| \leqslant 2 \varepsilon
$$

По теореме о непрерывной зависимости от начальных данных мы получаем утверждение леммы 1.

ЗАмЕчАНИЕ. Из доказательства следует, что мы можем обобщить утверждения леммы 1 и теоремы 2 на случай, когда $p+1=2^{*}-$ любое и вместо $u^{p}$ стоит достаточно гладкая функция $g$ с $g(v+w)-g(v)=f(v, w) w^{2}+p|v|^{p-1} v w$, где $f$ удовлетворяет (9).

Отдел теоретических проблем РАН

Поступило

31.10 .95

Исправленный вариант

18.03 .96

\section{СПИСОК ЦИТИРОВАННОЙ ЛИТЕРАТУРЫ}

1. Похожаев С. И. // Докл. АН СССР.. Т. 165. № 1. С. 36-39. 2. Bahri A., Lions P.-L. // C. R. Acad. Sci. Paris. Ser. 1. 1985. V. 301. P. 145-147. 3. Struwe M. // Manuscr. Math. 1980. V. 32. P. 335-364. 4. Bahri A., Berestycki H. // Trans. Amer. Math. Soc. 1981. V. 267. Р. 1-32. 5. Похожаев С. И. // Тр. МИАН. 1993. Т. 201. С. 324-341. 6. Петровский И. Г. Лекции по теории обыкновенных дифференциальных уравнений. М.: Наука, 1970. 7. Свешников А. Г., Тихонов А. Н. Теория функций комплексной переменной. М.: Наука. 8. Chandrasekhar S. An introduction to the study of stellar structure. N. Y.: Dover, 1957. 